OPEN ACCESS

Edited by:

Tibor Hortobágyi,

University of Debrecen, Hungary

Reviewed by:

Kazunori Sango,

Tokyo Metropolitan Institute of Medical

Science, Japan

Michael Henri Ossipov,

inVentiv Health Clinical, United States

*Correspondence:

Lakshmi Vas

Lakshmi@paincareindia.com

Specialty section:

This article was submitted to

Neurodegeneration,

a section of the journal

Frontiers in Neuroscience

Received: 21 September 2018

Accepted: 09 January 2019

Published: 05 February 2019

Citation:

Vas $L$ (2019) Commentary: Selective Fiber Degeneration in the Peripheral Nerve of a Patient With Severe Complex Regional Pain Syndrome.

Front. Neurosci. 13:19.

doi: 10.3389/fnins.2019.00019

\section{Commentary: Selective Fiber Degeneration in the Peripheral Nerve of a Patient With Severe Complex Regional Pain Syndrome}

\author{
Lakshmi Vas* \\ Ashirvad Institute for Pain Management and Research, Mumbai, India
}

Keywords: complex regional pain syndrome, ultrasound-guided dry needling, neuromyopathy, myofacial pain, myofacial trigger points, reflex sympathetic dystrophy

\section{A Commentary on}

Selective Fiber Degeneration in the Peripheral Nerve of a Patient With Severe Complex Regional Pain Syndrome

by Yvon, A., Faroni, A., Reid, A. J., and Lees, V. C. (2018). Front. Neurosci. 12:207. doi: 10.3389/fnins.2018.00207

Complex regional pain syndrome (CRPS), a chronic pain condition affecting the extremities, can severely affect the quality of life. Beyond a consensus that CRPS arises from damage to the central and peripheral nervous systems, its etiopathology is poorly understood. In a recent publication in Frontiers in Neuroscience, Yvon et al. (2018) examined radial, median, and ulnar nerve specimens from a CRPS-affected amputated limb and showed widespread (47-58\%) selective degeneration in the larger myelinated $\mathrm{A} \alpha$ fibers (motor/proprioception) and in groups of small unmyelinated $\mathrm{C}$ fibers (Remak bundles), while the smaller $\mathrm{A} \delta$ (pain/temperature) fibers were spared.

The authors postulated that large A $\alpha$ fiber degeneration could consequently affect the associated innervated muscles. These findings are of interest to us as they provide a mechanistic explanation for the profound structural disruption we have observed in the muscles of CRPS-affected limbs using musculoskeletal ultrasound (MSKUSG) (Vas and Pai, 2012, 2014, 2016b; Vas et al., 2013, 2016c, 2018; Pai and Vas, 2018). The sonographic signature of normal muscle is fairly distinct with a well-defined hyperechoic epimysium with an echolucent (dark) background with bright punctate and curvilinear echoes of the perimysium that appear as bright streaks [Figure 1A, left panels; (Walker et al., 2004)]. In contrast, MSKUSG of the CRPS muscle reliably shows an extreme increase in echogenicity ("snowstorm-like" appearance) that is indicative of muscle fibrosis (Reimers et al., 1993), with loss of muscle outlines [Figure 1A, right panels; (Vas et al., 2013)]. In severe cases, several muscles coalesce together to form one indistinct mass of hyperechogenecity (Figure 1A, left panels), with a marked loss of muscle bulk compared with the normal limb, as indicated by caliper measurements (Vas et al., 2016c). Histopathological changes in the muscle in CRPS have been previously reported (van der Laan et al., 1998; Hulsman et al., 2009), and other studies have shown a robust correlation between histological and sonographic changes in muscle in patients with inflammatory myopathy (Reimers et al., 1993; Pillen et al., 2009), suggesting that the observed sonographic changes in the muscles of CRPS patients (Vas et al., 2013, 2016c) may be accompanied by histological changes. 


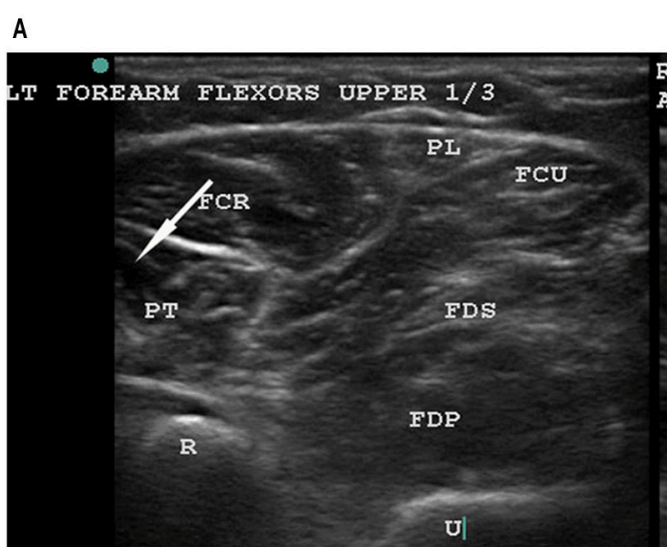

RT CRPS FOREARM UPPER $1 / 3$ FLEXORS. RADIUS

AT LOWER LEVEL THAN ULNA|

LT FOREARM EXTENSORS UPPER $1 / 3$

RT CRPS FOREARM UPPER $1 / 3$ EXTENSORS
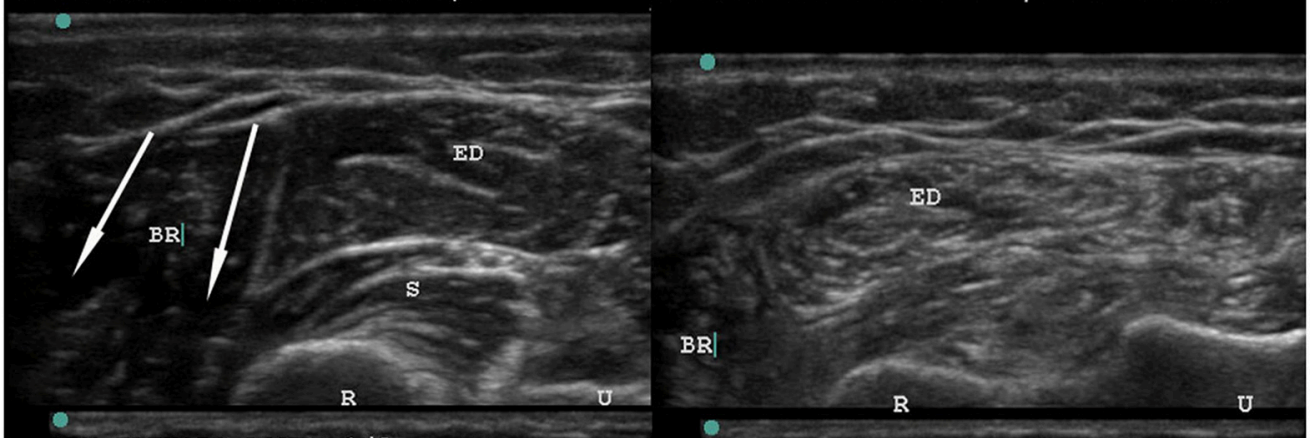

TT ARM FLEXORS LOWER $1 / 3$
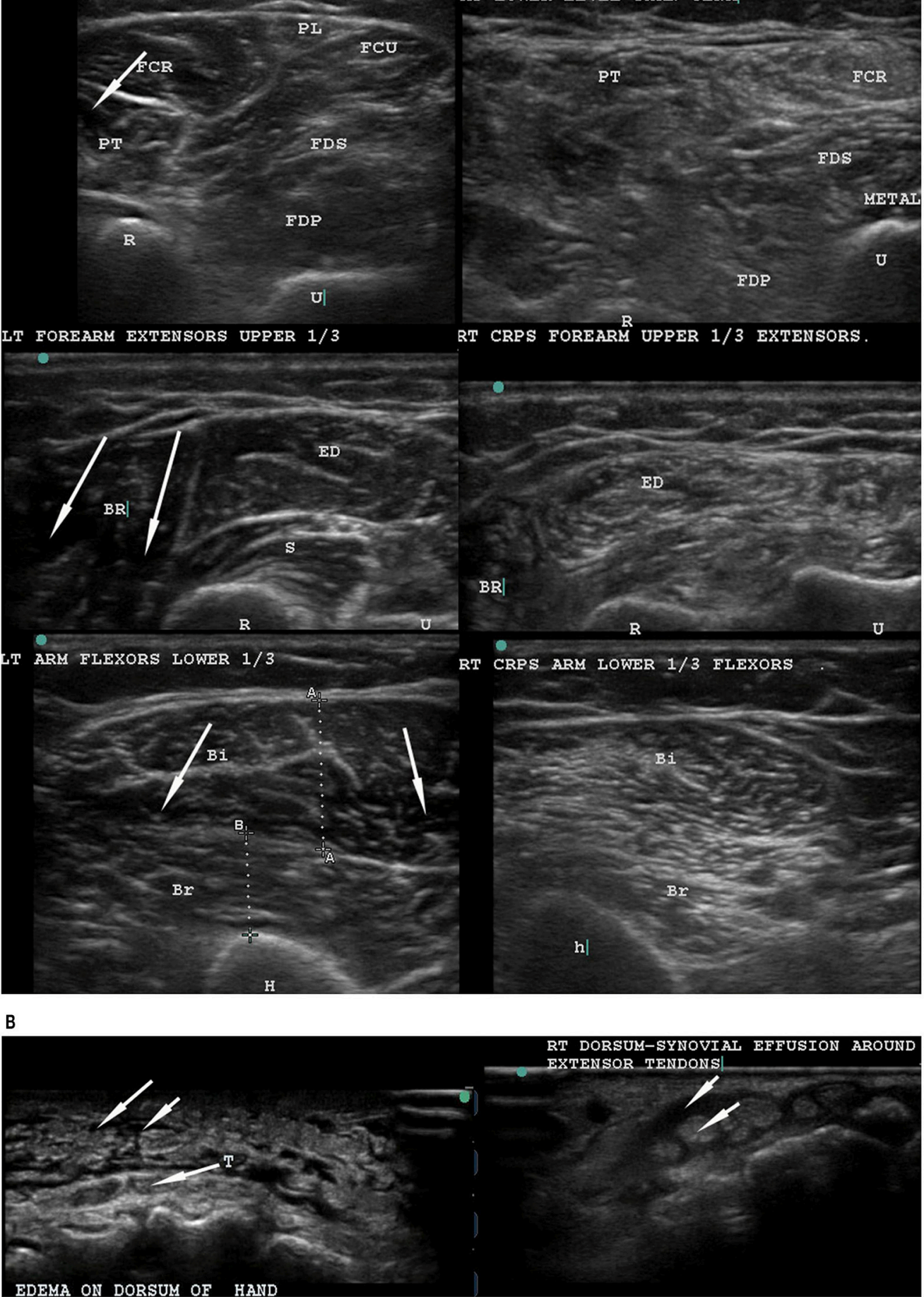

FIGURE 1 | Musculoskeletal ultrasonography to compare the normal left arm with the CRPS-affected right arm. (A) Musculoskeletal ultrasound of the normal left forearm (left) and the CRPS-affected right forearm (right) of a single patient. The normal left extremity muscles have well-defined outlines, and the muscle is hypoechoic (dark spaces, see arrows) with bright curvilinear echoes. In contrast, the CRPS-affected right arm and forearm muscles show general hyperechogenecity (as indicated by a snowstorm-like appearance not seen in normal muscle), with loss of the dark spaces as well as muscle outlines. (B) Ultrasonography of the dorsum of hand in a non-CRPS patient (left) and a CRPS patient (right). In the non-CRPS patient, the edema is subcutaneous (left, upper arrows) and superficial to the tendons (T) (arrow from T). In the CRPS-affected hand, a peritendinous tenosynovial effusion (right, upper arrow) surrounds the tendon (right, lower arrow). Note the normal narrow subcutaneous space without any fluid accumulation. T, tendon. 
Current treatments for CRPS such as neuromodulator pharmacotherapy and sympathetic ganglion block exclusively address nerve pathology, with limited results (Day, 2008; Gierthmühlen et al., 2014; Baig et al., 2017; Stanton-Hicks, 2018; Urits et al., 2018; Zyluk and Puchalski, 2018). Our approach has instead addressed muscle dysfunction through dry needling, a treatment where needles of $32-\mathrm{G}$ are placed at $1 \mathrm{~cm}$ intervals along both the length and breadth of muscles. Dry needling has been shown to deactivate MTrPs and relieve pain in a variety of conditions (Tsai et al., 2010; Mayoral et al., 2013; Rainey, 2013; Mejuto-Vázquez et al., 2014; Rock and Rainey, 2014; SalomMoreno et al., 2015; Shah et al., 2015). We have developed a more comprehensive and extensive dry needling protocol (discussed later), carried out under ultrasound guidance. As previously reported by our group (Vas and Pai, 2012, 2014, 2016a; Pai and Vas, 2018; Vas et al., 2018), this protocol of ultrasoundguided dry needling (USGDN) has shown remarkable success, producing not only sustained pain relief, but also disability relief, as indicated by improvement in the Disability of Arm, Hand, and Shoulder scale (Vas and Pai, 2016a,b). Importantly, USGDNtreated patients are able to routinely reassume their previous lifestyles and professions (Vas and Pai, 2012, 2014, 2016a; Vas et al., 2018).

We have also shown that this muscle fibrosis observed very consistently using MSKUSG in CRPS is not seen in other neuropathic pain conditions (Vas and Pai, 2016b). Strikingly, following CRPS reversal with USGDN, the profound structural disruption observable by MSKUSG is also reversed, with a return to normal muscle appearance (Vas et al., 2016c).

Based on findings of muscle changes and the routine reversal of muscle changes as well as CRPS symptoms, we propose that the primary pathology of CRPS is in the muscle, in the form of a co-contraction of the agonist and antagonist muscles responsible for performing the digital, wrist, elbow, and shoulder movements. We hypothesize that the abnormal co-activation of digital flexor-extensor and pronator-supinator muscles replaces the normal inbuilt reciprocal inhibition of agonist and antagonist muscles. Attempted movements of abnormally co-contracted muscles causes friction between the tendons and the tenosynovial sheaths, leading to the clinical presentation of tenosynovitis of all digital tendons. We have come to conclude that it is this very much exaggerated deQuervain-like involvement of all the flexor and extensor tendons that gives rise to all the manifestations of CRPS, including the sensory, motor, sudomotor, and vasomotor symptoms and signs (Reimers et al., 1993; van der Laan et al., 1998; Hulsman et al., 2009; Pillen et al., 2009; Vas and Pai, 2016b; Vas et al., 2016a,c). In fact, CRPS was mistakenly treated as deQuervain's tenosynovitis in a patient who later recovered completely after USGDN (Vas and Pai, 2016a). We have also

\section{REFERENCES}

Baig, S., Moon, J. Y., and Shankar, H. (2017). Review of sympathetic blocks: anatomy, sonoanatomy, evidence, and techniques. Reg Anesth Pain Med. 42, 377-391. doi: 10.1097/AAP.0000000000000591 found that MSKUSG clearly demonstrates the difference between tenosynovial effusion in the swollen CRPS hand (Figure 1B, left) from simple subcutaneous edema in a non-CRPS patient (Figure 1B, right).

USGDN is a simple yet effective technique that resolves the CRPS symptoms in a step-by-step manner. It would be easy for physicians to acquire the requisite skill by a thorough re-grounding in anatomy, sonoanatomy and the technique of needle placement in muscles under ultrasound guidance. However, USGDN reported in our publications should not be equated with the dry needling presently practiced in the US, Europe and elsewhere (Janz and Adams, 2011; Liu et al., 2015; Gattie et al., 2017; Tejera-Falcón et al., 2017; Rastegar et al., 2018). There are many important differences, including the number of needles (6 vs. 30-60 needles), length of needles (25-50 vs. $13-120 \mathrm{~mm})$, duration of needle maintenance in muscle ( $<1$ vs. $30 \mathrm{~min}$ ), maximum sessions (6 vs. 20), and method (blind unguided vs. ultrasound-guided). We believe that these differences, particularly ultrasound guidance, the number of needles, and duration of maintenance of needles in muscles, may contribute to the effectiveness of USGDN at our clinic in treating myriad conditions, including CRPS (Vas and Pai, 2012, 2014, 2016a; Vas et al., 2014a,b, 2015, 2016a,b,c, 2018).

In summary, we believe that the neural changes observed by Yvon et al. (2018) may be responsible for the muscle changes, but it is these muscle changes that form the primary pathology of CRPS. Indeed, degeneration of the larger $\mathrm{A} \alpha$ (motor/proprioception) fibers might be instrumental in producing the constant unrelenting muscle co-contraction that leads to generation of MTrPs. To our knowledge, no other groups have used MSKUSG to study CRPS, nor used USGDN as a specific treatment for CRPS. Exploration of these observations by other groups is necessary. Lastly, given the evidence implicating muscle pathology in CRPS, the suitability of USGDN (a remarkably safe technique) as a first-line option should be further explored, especially before considering more controversial approaches such as limb amputation.

\section{AUTHOR CONTRIBUTIONS}

The author confirms being the sole contributor of this work and has approved it for publication.

\section{ACKNOWLEDGMENTS}

Medical Writing services were provided by Jaya Vas Ph.D. of SimpliMed Writing and Editing Services, and were funded by Ashirvad Institute for Pain Management and Research.

Day, M. (2008). Sympathetic blocks: the evidence. Pain Pract. 8, 98-109. doi: 10.1111/j.1533-2500.2008.00177.x

Gattie, E. R., Cleland, J. A., and Snodgrass, S. J. (2017). Dry needling for patients with neck pain: protocol of a randomized clinical trial. JMIR Res Protoc. 6:e227. doi: $10.2196 /$ resprot.7980 
Gierthmühlen, J., Binder, A., and Baron, R. (2014). Mechanism-based treatment in complex regional pain syndromes. Nat Rev Neurol. 10, 518-528. doi: 10.1038/nrneurol.2014.140

Hulsman, N. M., Geertzen, J. H., Dijkstra, P. U., van den Dungen, J. J., and den Dunnen, W. F. (2009). Myopathy in CRPS-I: disuse or neurogenic? Eur J Pain 13, 731-736. doi: 10.1016/j.ejpain.2008.09.006

Janz, S., and Adams, J. (2011). Acupuncture by another name: dry needling in Australia. Aust J Acupunct Chin Med. 6, 3-11. Available online at: https://search.informit.com.au/documentSummary;dn=773412665943590; res=IELHEA

Liu, L., Huang, Q. M., Liu, Q. G., Ye, G., Bo, C. Z., Chen, M. J., et al. (2015). Effectiveness of dry needling for myofascial trigger points associated with neck and shoulder pain: a systematic review and meta-analysis. Arch Phys Med Rehabil. 96, 944-955. doi: 10.1016/j.apmr.2014.12.015

Mayoral, O., Salvat, I., Martín, M. T., Martín, S., Santiago, J., Cotarelo, J., et al. (2013). Efficacy of myofascial trigger point dry needling in the prevention of pain after total knee arthroplasty: a randomized, double-blinded, placebo-controlled trial. Evid Based Compl. Alternat Med. 2013:694941. doi: 10.1155/2013/694941

Mejuto-Vázquez, M. J., Salom-Moreno, J., Ortega-Santiago, R., TruyolsDominguez, S., and Fernandez-de-Las-Penas, C. (2014). Short-term changes in neck pain, widespread pressure pain sensitivity, and cervical range of motion after the application of trigger point dry needling in patients with acute mechanical neck pain: a randomized clinical trial. J Orthop Sports Phys Ther. 44, 252-260. doi: 10.2519/jospt.2014.5108

Pai, R. S., and Vas, L. (2018). Ultrasound-guided intra-articular injection of the radio-ulnar and radio-humeral joints and ultrasound-guided dry needling of the affected limb muscles to relieve fixed pronation deformity and myofascial issues around the shoulder, in a case of complex regional pain syndrome type 1. Pain Pract. 18, 273-282. doi: 10.1111/papr.12596

Pillen, S., Tak, R. O., Zwarts, M. J., Lammens, M. M., Verrijp, K. N., Arts, I. M., et al. (2009). Skeletal muscle ultrasound: correlation between fibrous tissue and echo intensity. Ultrasound Med Biol. 35, 443-446. doi: 10.1016/j.ultrasmedbio.2008.09.016

Rainey, C. E. (2013). The use of trigger point dry needling and intramuscular electrical stimulation for a subject with chronic low back pain: a case report. Int J Sports Phys Ther. 8, 145-161.

Rastegar, S., Baradaran Mahdavi, S., Hoseinzadeh, B., and Badiei, S. (2018). Comparison of dry needling and steroid injection in the treatment of plantar fasciitis: a single-blind randomized clinical trial. Int Orthop. 42, 109-116. doi: 10.1007/s00264-017-3681-1

Reimers, K., Reimers, C. D., Wagner, S., Paetzke, I., and Pongratz, D. E. (1993). Skeletal muscle sonography: a correlative study of echogenicity and morphology. J Ultrasound Med. 12, 73-77. doi: 10.7863/jum.1993. 12.2.73

Rock, J. M., and Rainey, C. E. (2014). Treatment of nonspecific thoracic spine pain with trigger point dry needling and intramuscular electrical stimulation: a case series. Int J Sports Phys Ther. 9, 699-711.

Salom-Moreno, J., Ayuso-Casado, B., Tamaral-Costa, B., Sánchez-Milá, Z., Fernandez-de-Las-Penas, C., and Alburquerque-Sendin, F. (2015). Trigger point dry needling and proprioceptive exercises for the management of chronic ankle instability: a randomized clinical trial. Evid Based Complement Alternat Med. 2015:790209. doi: 10.1155/2015/790209

Shah, J. P., Thaker, N., Heimur, J., Aredo, J. V., Sikdar, S., and Gerber, L. (2015). Myofascial trigger points then and now: a historical and scientific perspective. PM R. 7, 746-761. doi: 10.1016/j.pmrj.2015.01.024

Stanton-Hicks, M. (2018). "Complex regional pain syndrome," in Fundamentals of Pain Medicine, eds J. Cheng, and R. W. Rosenquist (Cleveland, $\mathrm{OH}$ : Springer), 211-220.

Tejera-Falcón, E., Toledo-Martel, N. D. C., Sosa-Medina, F. M., Santana-Gonzalez, F., Quintana-de la Fe, M. D. P., Gallego-Izquierdo, T., et al. (2017). Dry needling in a manual physiotherapy and therapeutic exercise protocol for patients with chronic mechanical shoulder pain of unspecific origin: a protocol for a randomized control trial. BMC Musculoskelet Disord. 18:400. doi: 10.1186/s12891-017-1746-3

Tsai, C. T., Hsieh, L. F., Kuan, T. S., Kao, M. J., Chou, L. W., and Hong, C. Z. (2010). Remote effects of dry needling on the irritability of the myofascial trigger point in the upper trapezius muscle. Am J Phys Med Rehabil. 89, 133-140. doi: 10.1097/PHM.0b013e3181a5b1bc

Urits, I., Shen, A. H., Jones, M. R., Viswanath, O., and Kaye, A. D. (2018). Complex regional pain syndrome, current concepts and treatment options. Curr Pain Headache Rep. 22:10. doi: 10.1007/s11916-018-0667-7

van der Laan, L., ter Laak, H. J., Gabreels-Festen, A., Gabreels, F., and Goris, R. J. (1998). Complex regional pain syndrome type I (RSD): pathology of skeletal muscle and peripheral nerve. Neurology 51, 20-25. doi: 10.1212/WNL.51.1.20

Vas, L., Khandagale, N., and Pai, R. (2014a). Successful management of chronic postsurgical pain following total knee replacement. Pain Med. 15, 1781-1785. doi: $10.1111 / \mathrm{pme} .12508$

Vas, L., and Pai, R. (2012). Successful reversal of complex regional pain syndrome type 1 of both upper extremities in five patients. Pain Med. 13, 1253-1256. doi: 10.1111/j.1526-4637.2012.01435.x

Vas, L., and Pai, R. (2014). Reversal of complex regional pain syndrome type 2 and the subsequent management of complex regional pain syndrome type 1 occurring after corrective surgery for residual ulnar claw. Pain Med. 15, 1059-1063. doi: 10.1111/pme.12381

Vas, L., and Pai, R. (2016a). Complex regional pain syndrome-type 1 presenting as dequervain's stenosing tenosynovitis. Pain Phys. 19, E227-E234. Available online at: http://www.painphysicianjournal.com/current/pdf?article=MjQ5OA \%3D\%3D\&journal=93

Vas, L., and Pai, R. (2016b). Musculoskeletal ultrasonography to distinguish muscle changes in complex regional pain syndrome type 1 from those of neuropathic pain: an observational study. Pain Pract. 16, E1-E13. doi: 10.1111/papr.12338

Vas, L., Pai, R., Geete, D., and Verma, C. V. (2018). Improvement in CRPS after deep dry needling suggests a role in myofascial pain. Pain Med. 19, 208-212. doi: $10.1093 / \mathrm{pm} / \mathrm{pnx} 124$

Vas, L., Pai, R., Khandagale, N., and Pattnaik, M. (2015). Myofascial trigger points as a cause of abnormal cocontraction in writer's cramp. Pain Med. 16, 2041-2045. doi: 10.1111/pme.12814

Vas, L., Pai, R., Pawar, K. S., and Pattnaik, M. (2016a). "Piriformis Syndrome”: Is It Only Piriformis? Pain Med. 17, 1775-1779. doi: 10.1093/pm/pnw037

Vas, L., Pattanik, M., and Titarmore, V. (2014b). Treatment of interstitial cystitis/painful bladder syndrome as a neuropathic pain condition. Indian J Urol. 30, 350-353. doi: 10.4103/0970-1591.128513

Vas, L., Phanse, S., and Pai, R. (2016b). A new perspective of neuromyopathy to explain intractable pancreatic cancer pains; dry needling as an effective adjunct to neurolytic blocks. Indian J Palliat Care 22, 85-93. doi: 10.4103/0973-1075.173957

Vas, L. C., Pai, R., and Pattnaik, M. (2016c). Musculoskeletal ultrasonography in CRPS: assessment of muscles before and after motor function recovery with dry needling as the sole treatment. Pain Phys. 19, E163-E179. Available online at: www.painphysicianjournal.com/current/pdf?article=MjQ5Mw\%3D $\% 3$ D\&journal $=93$

Vas, L. C., Pai, R., and Radhakrishnan, M. (2013). Ultrasound appearance of forearm muscles in 18 patients with complex regional pain syndrome 1 of the upper extremity. Pain Pract. 13, 76-88. doi: 10.1111/j.1533-2500.2012.00539.x

Walker, F. O., Cartwright, M. S., Wiesler, E. R., and Caress, J. (2004). Ultrasound of nerve and muscle. Clin Neurophysiol. 115, 495-507. doi: 10.1016/j.clinph.2003.10.022

Yvon, A., Faroni, A., Reid, A. J., and Lees, V. C. (2018). Selective fiber degeneration in the peripheral nerve of a patient with severe complex regional pain syndrome. Front Neurosci 12:207. doi: 10.3389/fnins.2018.00207

Zyluk, A., and Puchalski, P. (2018). Effectiveness of CRPS treatment: a systematic review. Neurol. Neurochir. Pol. 52:326-33. doi: 10.1016/j.pjnns.2018.03.001

Conflict of Interest Statement: The author declares that the research was conducted in the absence of any commercial or financial relationships that could be construed as a potential conflict of interest.

Copyright (C) 2019 Vas. This is an open-access article distributed under the terms of the Creative Commons Attribution License (CC BY). The use, distribution or reproduction in other forums is permitted, provided the original author(s) and the copyright owner(s) are credited and that the original publication in this journal is cited, in accordance with accepted academic practice. No use, distribution or reproduction is permitted which does not comply with these terms. 